\title{
How perception of personal space influence obstacle avoidance during walking: differences between young and older adults
} \author{
Taketo FurunA, PhD, PT $^{5}$ \\ ${ }^{1)}$ Graduate School of Health Sciences, Sapporo Medical University \\ ${ }^{2)}$ Department of Rehabilitation, Faculty of Health Sciences, Japan Health Care College \\ ${ }^{3)}$ Asahikawa Rehabilitation Hospital \\ ${ }^{4)}$ Houseikai Motomachi Himawari Clinic \\ ${ }^{5)}$ Department of Physical Therapy, School of Health Sciences, Sapporo Medical University
}

Kotaro ShIMIZU, Msc, PT ${ }^{1}$, Yuriko KIHARA, Msc, PT ${ }^{1,2}$, Kazunari ITOU, Msc, PT $^{3}$, Keita TAI, Msc, PT ${ }^{4}$ and

\begin{abstract}
Objective: Individuals maintain a spatial margin or 'personal space' between themselves and others. The form of this space and strategies for avoiding obstacles can be influenced by participant characteristics such as age. In this study, we investigated the characteristics of personal space and obstacle avoidance strategies in young and older adults. We also examined differences in perceptual personal space and walking trajectory during obstacle avoidance using a three-dimensional motion capture system. Methods Ten young adults and ten older adults participated in this study. We calculated actual obstacle avoidance trajectory and obstacle avoidance data such as the lateral spatial margin and body rotation angle during walking in a task that included obstacle avoidance. We also measured the perceptual personal space created by approaching a confederate. In order to calculate each personal space and obstacle avoidance data, we used a three-dimensional motion capture system. Two factors (two groups and personal space) of repeated analysis of variance were used in statistical analysis. Results We found no age-related differences in personal space or obstacle avoidance strategy in this study $(F=0.52, p=0.48)$. However, we found significant differences in the form of perceptual personal space and personal space formed during obstacle avoidance $(\mathrm{F}=$ 11.86, $p=0.0030$ ). Conclusion This study indicates that perceptual personal space did not reflect the walking trajectory created by actual obstacle avoidance. In addition, age did not influence the obstacle avoidance strategy. These results suggest that the perceptual personal space and aging have little effect in the situation of avoiding a single standing pedestrian.
\end{abstract}

Key words: older adults, obstacle avoidance, Personal Space

(Phys Ther Res 23: 31-38, 2020)

$\mathbf{W}_{\mathrm{h}}$ margin of space between themselves and other pedestrians, as well as obstacles, to avoid collisions ${ }^{1-3}$. Studies of adaptive gait have examined strategies for keeping spatial margins. These studies have shown body rotation and path deviation to be main obstacle avoidance strategies in situ-

Received: April 2, 2019

Accepted: October 5, 2019

Advance Publication by J-STAGE: February 25, 2020

Correspondence to: Kotaro Shimizu, Graduate School of Health Sciences, Sapporo Medical University, South1, West17 Chuo-ku, Sapporo, Hokkaido 060-8556, JAPAN

\# e-mail: shimizukotaro1009@gmail.com

doi: 10.1298/ptr.E9988 ations in which an individual passes through an aperture and during single obstacle avoidance ${ }^{1-4)}$. Hackney et al. investigated choices regarding obstacle avoidance strategies in the situation of passing through an aperture in various aperture width conditions ${ }^{5}$. They found that participants selected path deviation rather than body rotation when the aperture had narrow width. Other studies have investigated the relationship between obstacle width and individual variables, and reported that body scaled information such as an individual's shoulder width was important for decisions regarding obstacle avoidance strategy ${ }^{1,3,6)}$.

Adaptive obstacle avoidance strategies have been investigated using the concept of "personal space". This concept has been previously used in psychology research, and 
more recently as a measurement variable when examining obstacle avoidance strategies ${ }^{4,7)}$. Gelin-Lajoie et al. showed that personal space had an ellipsoidal form when participants avoided pedestrians while walking, and that personal space was wider in an anterior-posterior vs. medial-lateral direction $^{7}$. Therefore, individuals kept a wider anterior spatial margin and they maintained an anticipatory planned strategy of obstacle avoidance based on the area of personal space.

Kinematic and psychological studies have used different concepts to investigate an individual's spatial perception. Particularly, psychology studies have examined personal space according to self-body perception and the feeling of being close in proximity to other pedestrians ${ }^{8-10)}$. The perception of personal space has been found to be influenced by an individual's personal characteristics and environment. For instance, Adams et al. investigated the influence of brightness of environment and direction of approach on personal space ${ }^{8)}$. Zakay et al. also examined the influence of method of approach with respect to personal space $^{10)}$. Their data indicated that individuals maintained larger spatial margins when they were approached head-on by another pedestrian. Therefore, personal space measured in psychological studies is recognized as territory in which other pedestrians approach individuals.

Perception regarding interactions with other pedestrians is considered to require visual perception regardless of whether an individual avoids another pedestrian or recognizes the approach of another pedestrian. Previous studies have shown that vision is the only sensory function that can be used to interpret the distance between individuals and obstacles $^{11)}$. Therefore, vision is important in forming appropriate personal space, and visual impairment has been implicated in the use of abnormal obstacle avoidance strategies. Particularly, older adults have a higher risk of exhibiting abnormal obstacle avoidance strategies because they are more likely to receive incorrect visual information regarding the obstacle. In addition, declining physical function such as declining cognitive function in older adults has been considered a risk factor of collision with other pedestrians and failing to recognize spatial information of the environment, such as the presence of another pedestrian ${ }^{12)}$.

However, few studies have collected information regarding personal space formation in older adults. In particular, it is unclear how older adults, compared with young adults, keep a spatial margin to other pedestrians. In general, older adults have been found to be at a high risk of falling or collision in previous studies, and it has been considered that a poor ability to recognize spatial information is a factor responsible for falling and collision in older adults. It is thus important to clarify the relationship between physical instability caused by aging and spatial recognition in a situation of avoidance, such as that relating to personal space.
There is a difference between personal space created by the approaching pedestrian and personal space created by the actual avoidance trajectory. It is thus unknown whether perceptual personal space reflects actual obstacle avoidance in the situation of pedestrians approaching. If perceptual personal space affects the actual trajectory of obstacle avoidance, it is useful for the assessment of collision avoidance without actual walking assessment. Therefore, personal space is available for the safer assessment of highrisk individuals, such as those at high risk of falling and patients undergoing physical therapy. Moreover, the relationship between personal space and the actual avoidance strategy is important knowledge in studies of physical therapy, such as studies relating to the fall prevention of older adults and studies relating to the physical therapy of neurological disease.

In this study, we examined the perceptual personal space and actual avoidance strategy in the situation of passing through a pedestrian, and we compared the area of personal space and the actual trajectory of pedestrian avoidance in young and older adults. Additionally, we collected data regarding obstacle avoidance strategies, such as body rotation angle, to investigate the effect of aging on collision avoidance.

\section{Participants and Method}

\section{- Participants}

Ten young adults (three female, age: $21.6 \pm 1.6$ years, height: $170.0 \pm 9.9 \mathrm{~cm}$, weight: $60.1 \pm 11.0 \mathrm{~kg}$, shoulder width: $40.9 \pm 2.2 \mathrm{~cm}$ ) and ten older adults (five female, age: $75.6 \pm 5.0$ years, height: $155.8 \pm 9.2 \mathrm{~cm}$, weight: $57.2 \pm 12.3$ $\mathrm{kg}$, shoulder width: $41.0 \pm 3.0 \mathrm{~cm}$ ) participated in this study. This sample size was determined by the results of previous study ${ }^{13)}$. In calculating the sample size, we used $\mathrm{G}^{*}$ power 3.1.9.2. (Heinrich-Heine-Universität Düsseldorf, Germany). All participants were free from orthopedic or neurological disease that could influence obstacle avoidance. In addition, we tested the Mini-Metal-State-Examination before the examination to older adults' group in order to except cognitive impairment (mean score: $29.4 \pm 0.8$ ), and scores of all older adults were above 27 in Mini-Metal-StateExamination $^{14,15)}$. This study was approved by the Sapporo Medical University Ethics Committee (approval number: 28-2-26). All participants provided written informed consent, and the study was carried out according to the Helsinki Declaration.

\section{- Apparatus and procedure}

We collected data regarding perceptions of personal space, the relationship between personal space and obstacle avoidance, and obstacle avoidance strategies such as body rotation. All measurement variables were collected using the VICON motion capture system (Oxford Metrics, Ox- 


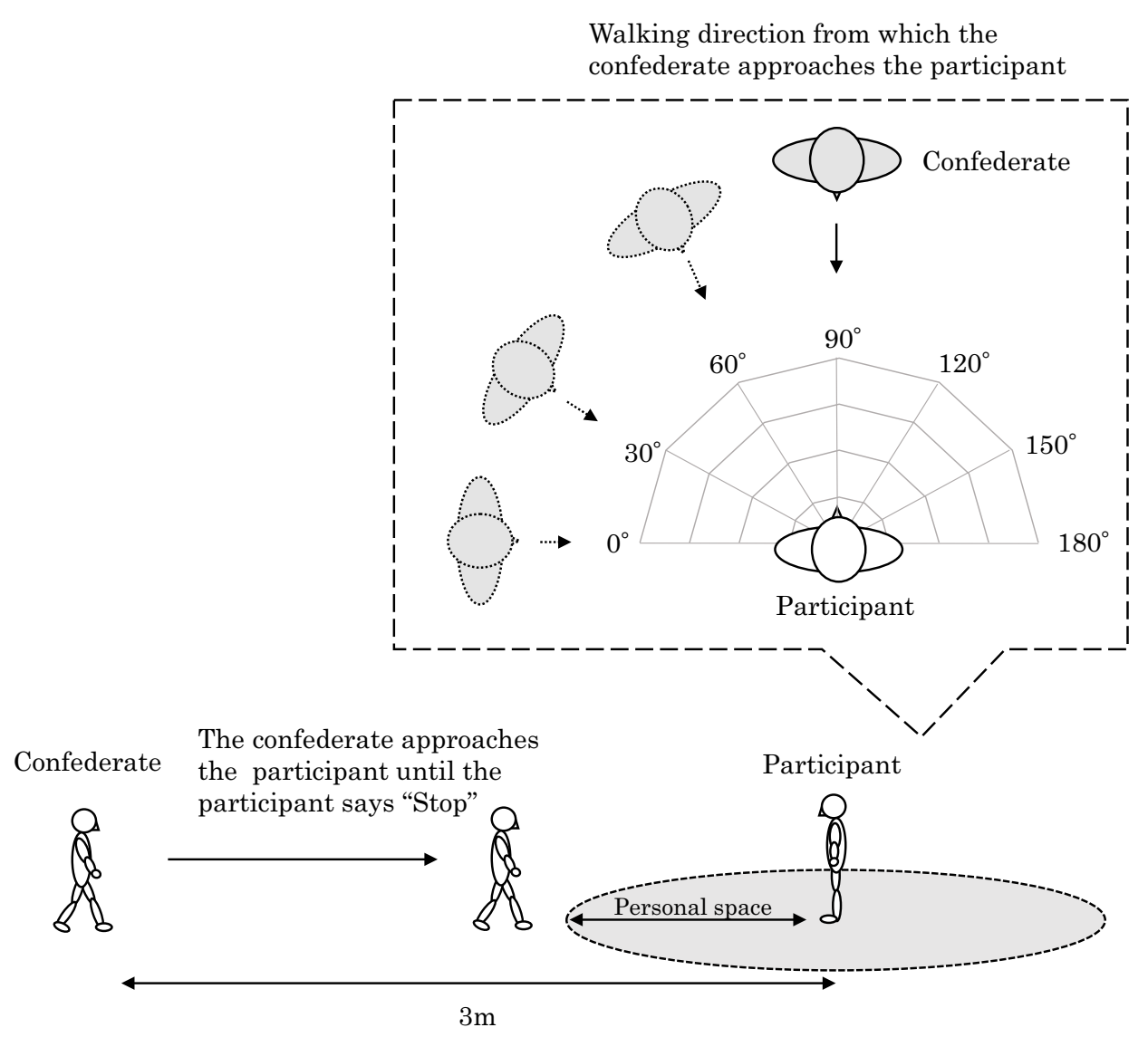

Figure 1. Calculation of perceptual personal space.

ford, UK). We positioned ten infrared cameras around a walkway. The cameras had a sampling rate of $100 \mathrm{~Hz}$. Sensors for the motion capture device were attached to the participant over the sternum and on each side of the shoulder (the lateral edge of acromion). Trials also included an experimenter who acted as a pedestrian. A sensor was attached to the pedestrian over the sternum.

\section{- Perceptual personal space}

We used a "stop distance procedure" to assess perceptions of personal space ${ }^{8)}$. This procedure is frequently used to assess personal space in psychological studies. In this method, an experimenter acting as a pedestrian approaches the participant, who is walking in a straight trajectory, from various walking directions. In this study, we used seven directions of approach of the pedestrian as follows (Figure 1): $0^{\circ}, 30^{\circ}, 60^{\circ}, 90^{\circ}, 120^{\circ}, 150^{\circ}$, and $180^{\circ}$. Participants were asked to report the distance at which they felt uncomfortable about the proximity of the approaching pedestrian for each walking direction. We performed three trials for each walking direction condition. When approaching the pedestrian, participants were asked not to rotate their head or trunk.

For each walking direction, we represented the area corresponding with personal space as a triangle with two sides as the two walking trajectories joined by a side corre- sponding to the distance at which discomfort was reported.

\section{- Actual trajectory of pedestrian avoidance}

We examined pedestrian avoidance strategies during walking. Participants were instructed to avoid a confederate standing $6 \mathrm{~m}$ from the start point in a $10 \mathrm{~m}$ walking path. The pedestrian was the same person in all trials (height: $162.0 \mathrm{~cm}$, weight: $53.0 \mathrm{~kg}$, shoulder width: $38.5 \mathrm{~cm}$ ).

We asked participants to walk normally and avoid the confederate if necessary. The avoidance direction was defined as either leftward or rightward, and participants were asked to avoid making a decision about which direction to choose prior to the trial. We conducted 3 trials for each avoidance direction. To compare actual trajectories of pedestrian avoidance, we calculated triangular areas formed by the trajectory of pedestrian avoidance and the minimum distances between the pedestrian and participant at angles of $30^{\circ}, 60^{\circ}$, and $90^{\circ}$ to the walking path and then summed triangular areas as shown in Figure 2.

\section{- Body rotation angle and lateral spatial margin}

We calculated 1) the body rotation angle and 2) the lateral spatial margin as obstacle avoidance strategies. The body rotation angle was calculated via markers attached to each shoulder of the participant at the moment that the participant passed the pedestrian. Lateral spatial margin was 
An example of the triangle formed from the minimum distance between the participant and obstacle

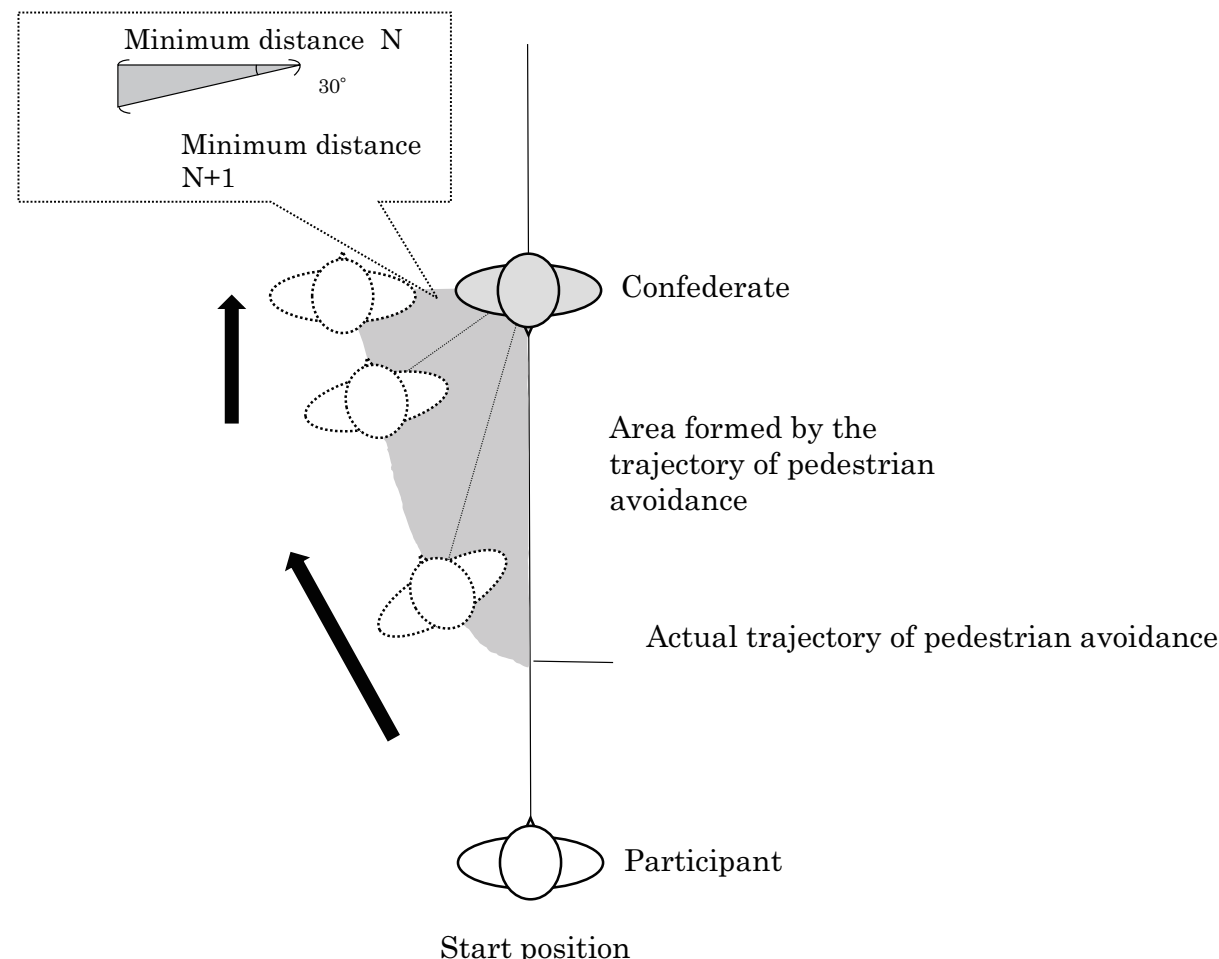

Figure 2. Calculation of area formed by the trajectory of pedestrian avoidance.

calculated as the distance between the sternum markers on the participant and confederate at the moment that the participant passed pedestrian.

\section{- Statistical analysis}

To investigate the influence of aging and the method of measuring personal space, we used a two-way ANOVA with repeated measures. We also used a two-way repeated ANOVA ( two avoidance direction $\times$ two participants groups) to examine body rotation and the lateral spatial margin. About relationship between perceptual personal actual, trajectory of pedestrian avoidance and shoulder width, Pearson's correlation analysis was used in order to investigate the difference of shoulder width in each participant. A $p$ value of less than 0.05 was defined as statistically significant. All statistical analyses were performed using SPSS version 20 (IBM Japan Ltd, Tokyo Japan).

\section{Results}

\section{- Personal space and actual pedestrian avoidance trajec-} tory

The means and standard deviations of personal space for each group and measurement method are shown in Figure 3 and Figure 4.

The two-way repeated ANOVA showed a main effect of measurement method $(F=11.86, p=0.0030)$. This result indicates that personal space formed during obstacle avoid- ance was larger than perceptual personal space. The main effect of aging $(F=0.52, \quad p=0.48)$ and the interaction effect $(\mathrm{F}=2.65, \mathrm{p}=0.12)$ were not significant, indicating that personal space did not vary among the participant groups.

\section{- Body rotation angle and lateral spatial margin}

The means and standard deviations of the body rotation angle and lateral spatial margin are shown in Figure 5 and Figure 6.

We found no main effects of avoidance direction $(\mathrm{F}=$ $0.023, \mathrm{p}=0.88)$ or aging $(\mathrm{F}=0.28, \mathrm{p}=0.60)$, and no interaction effect $(\mathrm{F}=0.060, \mathrm{p}=0.81)$. These results indicate that body rotation angle and lateral spatial margin were not affected by age or avoidance direction.

- The relationship between perceptual personal space, trajectory of pedestrian avoidance, and shoulder width

Results of Pearson's correlation analysis, there were no significant correlation between perceptual personal space and shoulder width in each participant's group (young adults group: $\mathrm{r}=-0.38, \mathrm{p}=0.39$, older adults group: $r=0.26, p=0.48)$. Similarly, there was no were no significant correlation between trajectory of pedestrian avoidance and shoulder width in each participant's group (young adults group: $\mathrm{r}=-0.54, \mathrm{p}=0.10$, older adults group: $r=-0.065, p=0.86)$. These results indicated that shoulder width had little association with perceptual personal space and trajectory of pedestrian avoidance. 


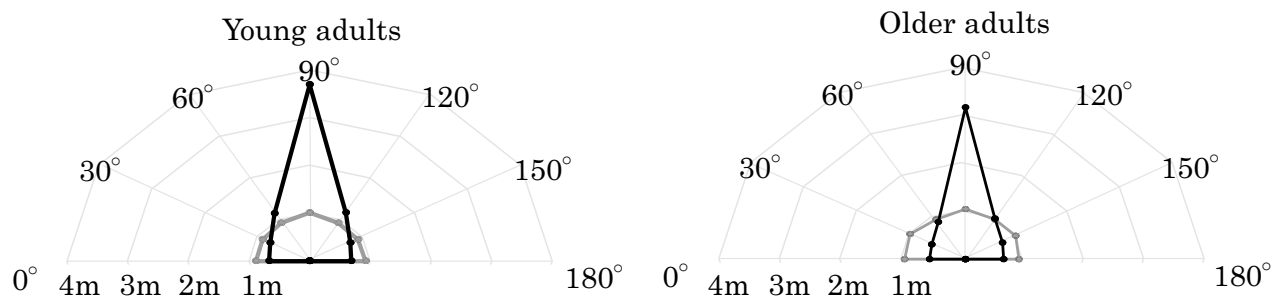

The distance between participant and confederate in each direction.

The distance between participant and confederate in each direction.
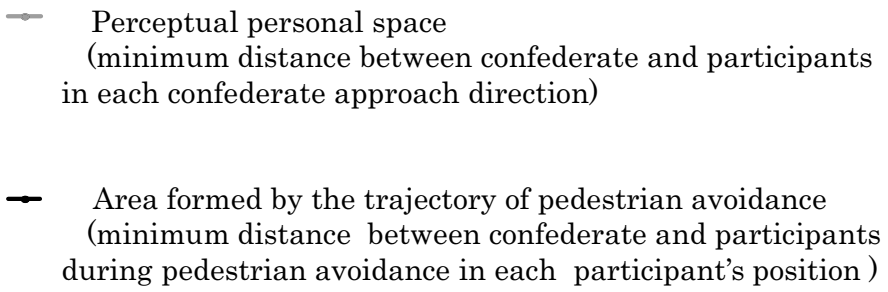

Figure 3. Mean perceptual personal space and area formed by the trajectory of pedestrian avoidance in each participant group.

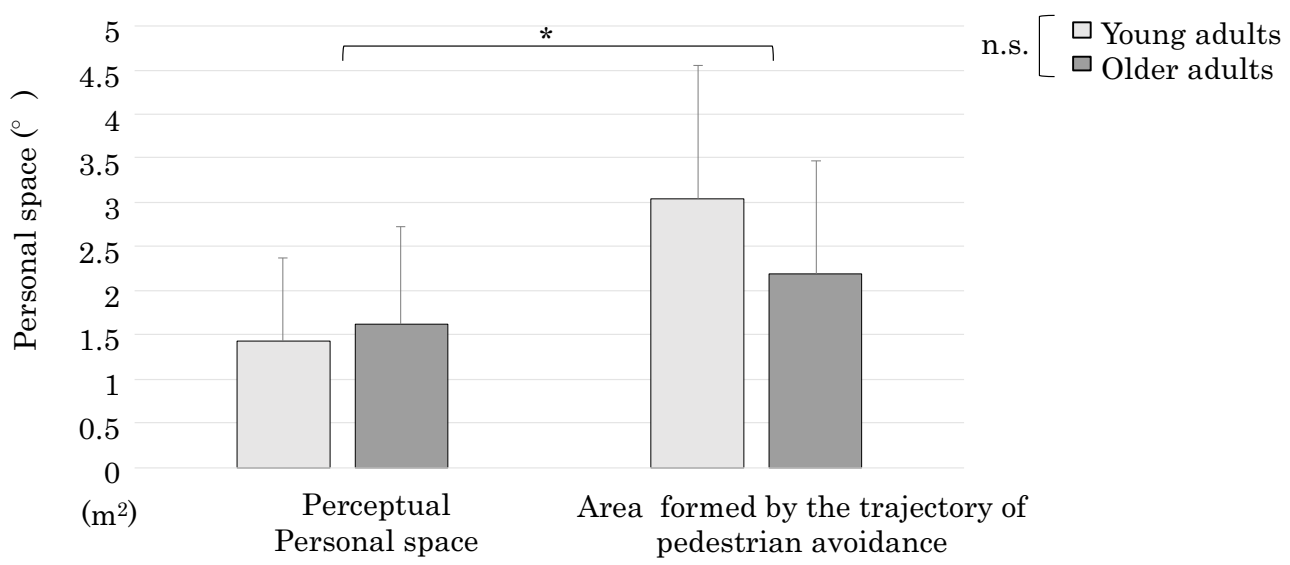

Figure 4. The means and standard deviation of perceptual personal space and area formed by the trajectory of pedestrian avoidance.

$(*$ p value was less than 0.05 in the main effect of two-way repeated ANOVA)

(n.s. = p values was 0.05 or more in the main effect of two-way repeated ANOVA)

\section{Discussion}

Our data indicate that personal space can vary significantly depending on the measurement method used. Additionally, we found that age did not influence the area of personal space.

As for collision avoidance strategy, we found no differences according to age or avoidance direction. In addition, perceptual personal space and trajectory of pedestrian avoidance were not influenced by body- build in each subject.

- Perceptual personal space vs. actual pedestrian avoidance trajectory

Our results indicate that the actual avoidance trajec- tory was wider than the perceptual personal space. We found an especially large difference in the anterior region of the personal space area. Previous studies that investigated personal space during walking have reported similar results $^{4,7,13)}$. For instance, Gelin-Lajoie et al. examined personal space in individual pedestrians, and reported that the personal space of participants formed an ellipse with longitudinal and lateral axes equal to $2.11 \mathrm{~m}$ and $0.48 \mathrm{~m}$, on average $^{7)}$. The size and shape of the personal space area in the present study was similar to that of previous studies.

As for perceptual personal space, our data were consistent with those of previous studies. Zukay et al. examined perceptual personal space from a psychological perspective. Specifically, they measured the minimum distance at which participants felt uncomfortable regarding a pedes- 

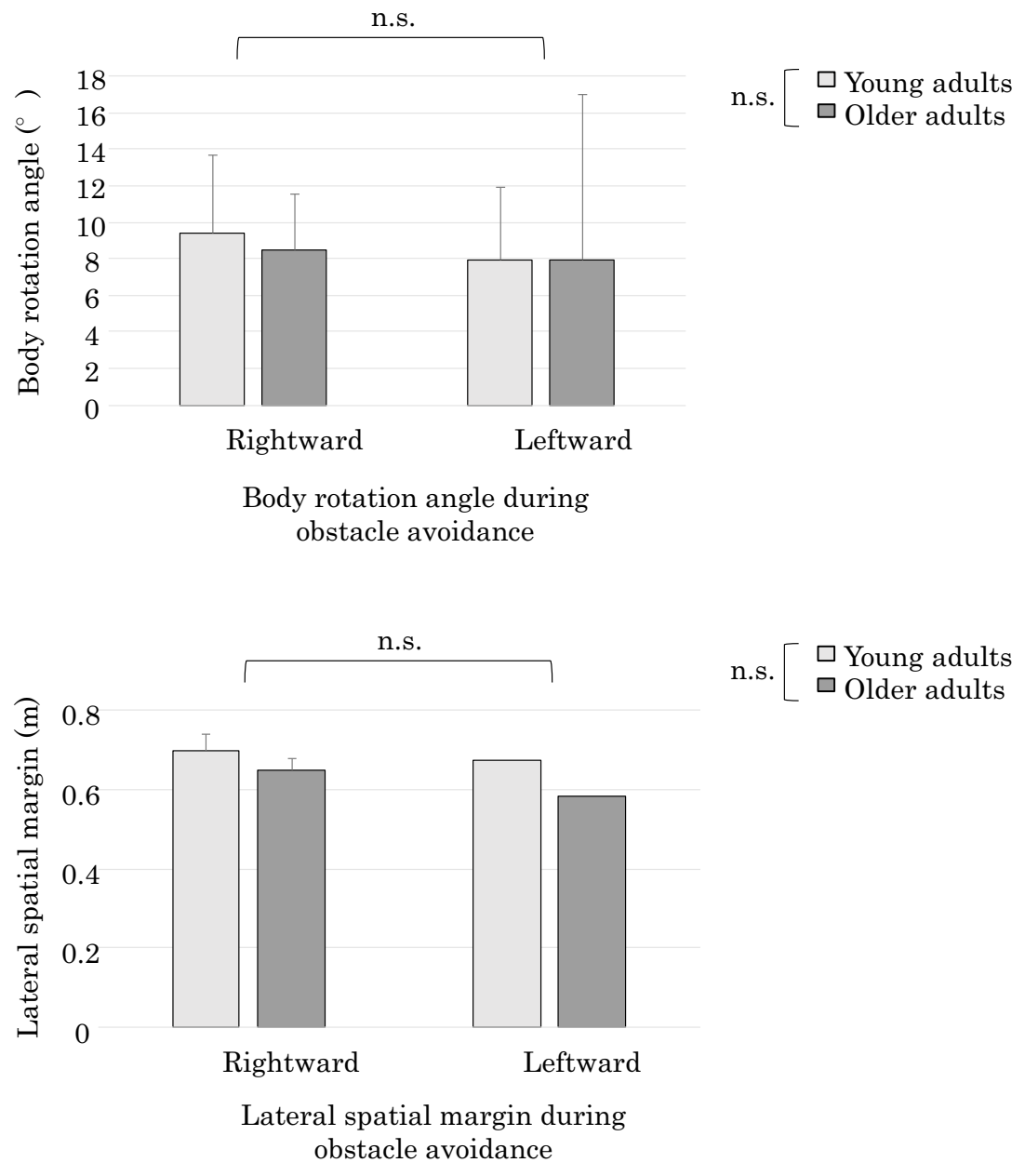

Figure 5. The means and standard deviation of body rotation angle and lateral spatial margin during obstacle avoidance in each age group. (n.s. = p values was 0.05 or more in the main effect of two-way repeated ANOVA)

trian approaching from a head-on direction. Similar to the present study, the researchers found the mean distance at which participants felt uncomfortable to be $1.77 \pm 0.4 \mathrm{~m}^{10)}$. Adams et al. examined the area of personal space from a psychological perspective. Specifically, they examined the characteristics of personal space areas formed in bright or dimly lit environments, and found that in both environments, participants formed a round-shaped personal space area ${ }^{8)}$. This is consistent with the findings of the present study.

The personal space data collected in the present study are consistent with previous findings. This suggests that the perceptual personal space and actual pedestrian avoidance trajectory are fundamentally different. In other words, perceptual personal space did not reflect actual obstacle avoidance strategies. This is likely because individuals-initiated obstacle avoidance quickly to avoid the discomfort caused by approaching the confederate. Therefore, compared with the distance required to successfully avoid an obstacle, individuals require a greater distance between themselves and others to feel comfortable.

- The influence of aging on the form of the personal space area

In this study, we found no differences in the personal space area and actual pedestrian avoidance trajectory between young and older adults. Previous studies have shown that young and older adults use different obstacle avoidance strategies $^{8,12,16-18)}$. For instance, Hackney et al. investigated obstacle avoidance strategies as participants passed through an aperture, and found that older adults tended to go around the aperture regardless of whether the aperture was sufficiently wide to pass through ${ }^{12}$. Furthermore, differences in visual sampling during adaptive gait have been found in adults vs. young adults ${ }^{16,17)}$.

Generally, aging is considered to be a major cause of decreased physical function, for instance, impaired gait, and reduced cognitive function ${ }^{19-23)}$. Executive function and attentional function are known to be important for perceiving the environment with respect to adaptive gait ${ }^{19,23)}$. 
Contrary to the results of previous studies, we found no differences in the personal space area and actual pedestrian avoidance trajectory between older and young adults regardless of age affecting physical function. In this study, participants in the older group retained good cognitive function. There was thus less of an effect of reduced cognitive function due to aging in perceiving spatial information of the obstacle.

\section{- Characteristics of obstacle avoidance strategies in each participant group}

Contrary to previous studies, we found no differences in the collision avoidance strategies used by older and young adults ${ }^{16-18,24,25)}$. Previous studies have investigated obstacle avoidance strategies while older adults walked in various environments. These studies have indicated that deficits in physical function, such as those arriving from cognitive impairments, affect the selection of obstacle avoidance strategies. Compared with young adults, older adults are more likely to select strategies that are safer, especially older adults who are prone to falling. However, the present data showed no effect of aging on any obstacle avoidance strategies or method of personal space formation.

In the present study, cognitive function (MMSE 27>) and vision were equal in the older and younger adult groups. This result suggests that obstacle avoidance strategies are influenced by the physical function of an individual. In other words, older adults appear to use perceptual information and cognitive processing when performing motor controls associated with obstacle avoidance. Therefore, assessing age-related conditions that could affect physical function such as dementia and sarcopenia may be helpful in screening for individuals who may engage in adaptive locomotor control when in a crowd.

Although the selection of obstacle avoidance strategies did not appear to differ in older vs. younger adults in the present study, the selection of obstacle avoidance strategies has been previously found to depend on the environment and individual participant characteristics ${ }^{2,3,7,24,25}$. For instance, in studies that included an aperture condition, individuals were found to rotate their shoulders, while individuals were more likely to select path deviation as an obstacle avoidance strategy when there was a single obstacle located on a pathway. The present data indicate that aging did not influence the selection of obstacle avoidance, and that individuals prefer to select path deviation rather than body rotation during single confederate avoidance.

\section{- The influence of avoidance direction}

In addition to the influence of aging on the form of personal space and obstacle avoidance strategy, we investigated the influence of avoidance direction on the obstacle avoidance strategy. Previous studies have investigated the laterality of the spatial margin at the moment of obstacle avoidance, ${ }^{26,27)}$ and found that individuals had a laterality. Specifically, individuals tended to maintain a wider space on the left side.

Contrary to previous studies, we found no influence of avoidance direction on body rotation angle or lateral spatial margin. It is possible that individual differences among the participants produced a high variation in obstacle avoidance strategies. The laterality of obstacle avoidance strategies is thought to be influenced by participant characteristics such as handedness and dominant eye ${ }^{28,29)}$. However, the relationships between these characteristics have not yet been determined. It appears that obstacle avoidance strategies are dependent on individual differences, with no common stereotypes.

\section{Limitation}

In the present study, older participants had no cognitive impairment, such as dementia or mild cognitive impairment. The effects of cognitive function on the perception of a pedestrian are unknown. Moreover, participants were only tested in terms of their perceptual personal space and actual pedestrian avoidance trajectory, and we did not test physical functionality, such as the ability to walk fast and with balance. Accordingly, there is the possibility that the group of older adults still had good physical functionality. Furthermore, the experimental environment of this study differed from a real-life environment such as a crowded station platform. It is therefore unclear whether the relationship between the personal space and actual obstacle avoidance applies to real-life situations.

\section{Conclusion}

In the present study, we investigated the influence of aging on the form of personal space and obstacle avoidance strategies during a task in which a participant walked towards a single confederate standing on a pathway. Our results showed no differences in personal space or obstacle avoidance strategy between older and younger adults. In addition, perceptual personal space did not reflect the actual obstacle avoidance trajectory in either participant group. Generally, individuals appear to maintain a wider spatial margin when they feel uncomfortable regarding the distance between themselves and a confederate.

Acknowledgments: This study was funded by Japanese Physical Therapy Association. We thank Sydney Koke, MFA, from Edanz Group (www.edanzediting.com/ac) for editing a draft of this manuscript. We also thank Prof. Takahiro Higuchi from Tokyo Metropolitan University for his valuable comments.

Conflict of Interest: There is no conflict of interest to 
disclose.

\section{References}

1) Hackney A. L., Cinelli M. E., et al.: Does the passability of apertures change when walking through human versus pole obstacles? Acta Psychol (Amst). 2015; 162: 62-68.

2) Vallis L. A. and McFadyen B. J.: Locomotor adjustments for circumvention of an obstacle in the travel path. Exp Brain Res. 2003; 152: 409-414.

3) Warren W. H. and Whang S.: Visual guidance of walking through apertures: body-scaled information for affordances. J Exp Psychol Hum Percept Perform. 1987; 13: 371-383.

4) Fajen B. R. and Warren W. H.: Behavioral dynamics of steering, obstacle avoidance, and route selection. J Exp Psychol Hum Percept Perform. 2003; 29: 343-362.

5) Hackney A. L., Cinelli M. E., et al.: Is the critical point for aperture crossing adapted to the person-plus-object system? J Mot Behav. 2014; 46: 319-327.

6) Hackney A. L., Vallis L. A., et al.: Action strategies of individuals during aperture crossing in nonconfined space. Q J Exp Psychol (Hove). 2013; 66: 1104-1112.

7) Gérin-Lajoie M., Richards C. L., et al.: The circumvention of obstacles during walking in different environmental contexts: a comparison between older and younger adults. Gait Posture. 2006; 24: 364-369.

8) Adams L. and Zuckerman D.: The Effect of Lighting Conditions on Personal Space Requirements. The Journal of General Psychology. 1991; 118: 335-340.

9) Kaya N. and Erkip F.: Invasion of personal space under the condition of short-term crowding: A case study on an automatic teller machine. Journal of Environmental Psychology. 1999; 19: 183-189.

10) Zakay D., Hayduk L. A., et al.: Personal space and distance misperception: Implications of a novel observation. Bulletin of the Psychonomic Society. 1992; 30: 33-35.

11) Patla A. E. and Vickers J. N.: Where and when do we look as we approach and step over an obstacle in the travel path? Neuroreport. 1997; 8: 3661-3665.

12) Hackney A. L. and Cinelli M. E.: Older adults are guided by their dynamic perceptions during aperture crossing. Gait Posture. 2013; 37: 93-97.

13) Gérin-Lajoie M., Richards C. L., et al.: The negotiation of stationary and moving obstructions during walking: anticipatory locomotor adaptations and preservation of personal space. Motor Control. 2005; 9: 242-269.

14) Bour A., Rasquin S., et al: How predictive is the MMSE for cognitive performance after stroke? Journal of neurology. 2010; 257: 630-637.

15) Markwick A., Zamboni G., et al.: Profiles of cognitive subtest impairment in the Montreal Cognitive Assessment (MoCA) in a research cohort with normal Mini-Mental State Examination (MMSE) scores. Journal of clinical and experimental neuropsychology. 2012; 34: 750-757.

16) Chapman G. J. and Hollands M. A.: Evidence for a link between changes to gaze behaviour and risk of falling in older adults during adaptive locomotion. Gait Posture. 2006; 24: 288-294.

17) Chapman G. J. and Hollands M. A.: Evidence that older adult fallers prioritise the planning of future stepping actions over the accurate execution of ongoing steps during complex locomotor tasks. Gait \& posture. 2007; 26: 59-67.

18) Chapman G. J. and Hollands M. A.: Age-related differences in visual sampling requirements during adaptive locomotion. Exp Brain Res. 2010; 201: 467-478.

19) Amboni M., Barone P., et al.: Cognitive contributions to gait and falls: evidence and implications. Movement disorders: official journal of the Movement Disorder Society. 2013; 28: 15201533.

20) Beauchet O., Annweiler C., et al.: Stops walking when talking: a predictor of falls in older adults? European journal of neurology. 2009; 16: 786-795.

21) Beauchet O., Dubost V., et al.: Dual-task-related gait changes in transitionally frail older adults: the type of the walkingassociated cognitive task matters. Gerontology. 2005; 51: 48-52.

22) Lundin Olsson L., Nyberg L., et al.: "Stops walking when talking" as a predictor of falls in elderly people. The Lancet. 1997; 349: 617.

23) Yogev-Seligmann G., Hausdorff J. M., et al.: The role of executive function and attention in gait. Movement disorders: official journal of the Movement Disorder Society. 2008; 23: 329-342; quiz 472.

24) Hackney A. L. and Cinelli M. E.: Action strategies of older adults walking through apertures. Gait Posture. 2011; 33: 733736.

25) Hackney A. L. and Cinelli M. E.: Young and older adults use body-scaled information during a non-confined aperture crossing task. Exp Brain Res. 2013; 225: 419-429.

26) Moussaïd M., Helbing D., et al.: Experimental study of the behavioural mechanisms underlying self-organization in human crowds. Proc Biol Sci. 2009; 276: 2755-2762.

27) Nicholls M. E., Loftus A., et al.: Things that go bump in the right: The effect of unimanual activity on rightward collisions. Neuropsychologia. 2007; 45: 1122-1126.

28) Fujikake H., Higuchi T., et al.: Directional bias in the body while walking through a doorway: its association with attentional and motor factors. Experimental brain research. 2011; 210: 195-206.

29) Kitayama S., Fujikake H., et al.: The relationship between spatial cognition and walking trajectory for passing through a doorway: Evident in individuals with dominant right eye? Experimental brain research. 2015; 233: 797-807. 